\title{
Interference Localization On-Board the Satellite Using Drift Induced Virtual Array
}

\author{
Aakash Arora*, Sina Maleki*, Bhavani Shankar Mysore Rama Rao*, Joel Grotz ${ }^{\dagger}$, and Björn Ottersten* \\ ${ }^{*}$ SnT - Interdisciplinary Centre for Security, Reliability and Trust \\ University of Luxembourg, Luxembourg \\ Email: \{aakash.arora, sina.maleki, bhavani.shankar, bjorn.ottersten\} @uni.lu \\ †SES Engineering, System Architecture \& Innovation, Luxembourg \\ Email: Joel.Grotz@ses.com
}

\begin{abstract}
Herein, we investigate the interference received from other wireless networks into a satellite communication (SATCOM) link, and review approaches to identify the interference location using on-board satellite processing. Interference is an increasing problem for satellite communication links, and while receiving signals from gateways or user terminals, the uplink is prone to disturbance by interference due to jammers or unintentional transmissions. In this paper, our aim is to localize unknown interference sources present on the ground by estimating direction of arrival (DOA) information using onboard processing (OBP) in the satellite, and the satellite drift inducing a virtual array. In this work, the signal sampled by the drifting single antenna feed is modeled as using an arbitrary array. Building on this model, we perform the 2-D DOA (azimuth and elevation) estimation. The key challenges in such a design include single snapshot based DOA estimation with low complexity and robustness, arising out of limited on-board computational complexity as well as uncertainty in parameters like the drift speed. Employing realistic satellite drift patterns, the paper illustrates the performance of the proposed technique highlighting the accuracy in localization under adverse environments. We provide numerical simulations to show the effectiveness of our methodology.
\end{abstract}

\section{INTRODUCTION}

In a SATCOM system, inter-system interference due to jammers, misaligned dish antennas etc., has become an important concern. With the rise of digital media, mobile network services etc., the geostationary orbit is becoming crowded [1],[2], and the amount of interference experienced by the satellite system keeps on increasing. The interference substantially deteriorates the signal quality and thus degrades the overall system throughput, and performance. In general, intersystem interference can be intentional, which is caused by jammers etc., or unintentional from misaligned dish antennas, equipment mismatch, etc. Especially, with an increasing number of satellite user terminals, the interference caused by misaligned antennas has become a serious problem for the SATCOM operators, and service providers [3]. In turn, this affects the quality of service and revenue. Therefore, localization is essential to handle the interference and effective measures should be taken for its mitigation.

Localizing an interfering station provides the necessary information to mitigate the ambient interference and further, prevent such events happening in the future. There have been various techniques which uses TDOA, FOA, and FDOA
[2], [4], [5] based measurements to localize an interference using the ground equipment for geostationary (GEO) satellite systems. But most of the methods operate on-ground and require access to multiple satellites receiving the same interference, which might not be possible in practice. Recent attempts have also been made to localize interfering signals using a satellite [2], [6], [5], such as frequency of arrival (FOA) based interference localization can be performed using single satellite [5]. But these methods can localize at most one interference. In other words, existing interference localization techniques employ on-ground methods, wherein, the interference corrupted signals from one or more satellites are processed on the ground. Moreover, the existing solutions do not provide reliable localization accuracy, owing to the impact of propagation losses. This has encouraged the satellite industry to look into employing on-board single satellite localization solutions to improve the localization accuracy in all situations irrespective of the number of available satellites. Single satellite-based interference techniques will be feasible in future because of the advancements in satellite payload to provide OBP facilities. Therefore, the ultimate aim is to employ single satellite-based localization schemes on-board the satellite.

On-board power limitation and lower computational complexity are the key challenges, which should be taken into consideration while providing the solution. On-board localization provides some advantages like there is no need to relay signals to the gateway $(\mathrm{GW})$, no large antenna gain is required to reduce the uplink noise. In this work, we present single feed of a satellite-based DOA localization of the interferer on-board the satellite. For the worst case design, we consider interfering signal to be in the same frequency band with the uplink. Single feed scenario is taken into the model, to keep computational complexity small. The range of the interfering signal is the earth radius because it is present on the earth's surface. That is why we prefer to perform DOA based localization. Thus, azimuth and elevation are the only parameters to estimate in the spherical coordinate system. It is well known that for DOA estimation one requires an array. Moreover, to estimate 2-D DOA, we require a 2-D or 3-D array.

A geostationary (GEO) satellite drifts from its position on a daily basis because of the gravitational influence from the sun, 
and the moon [1]. This phenomenon results in a displacement of the satellite from its actual position. More information regarding the roles of the earth's gravitational forces, third body (Moon and Sun) effects, trajectory or drifting pattern can be found in [7], [8]. In [7] authors explain that for a GEO satellite, periodically station keeping maneuvers need to be performed to correct the satellite position. This procedure uses resources like fuel, which essentially reduces the lifetime of the satellite. But from an interference localization point-of-view, this drift can be utilized to create a virtual or synthetic aperture array for localization. To be more specific, we consider the drifting single feed case which creates a drift induced virtual arbitrary sensor array. Although, we have a drift induced virtual array (DIVA) still the localization problem is difficult because we consider a single snapshot case. On-board processing (OBP) provides the ability to sample at a higher rate than the station keeping intervals. Therefore the problem is to estimate the DOA using a high dimensional spatial snapshot corresponding to a large number of sensors. Here, we provide a solution to this problem by transforming a very long measurement vector to the multiple snapshots.

The paper is organized into several sections: section II presents the system model, section III presents the on-board interference localization methodology adopted to provide the solution. Then we provide numerical simulation results in section IV to illustrate the feasibility of the method and finally, we conclude by providing a summary of the paper. In future, we would like to find theoretical guarantees under which proposed solution works.

\section{System Model}

In a satellite system, multiple feeds are employed in the uplink to receive signals from the $\mathrm{GW}$, and an uplink interference can completely block the link, thereby interrupting the transmission of signals from a GW to the satellite, or from the user terminals to the satellite. In order to localize an interferer on-board the satellite, we utilize the satellite drift to create a DIVA which possibly can localize the interference. To do this, we make use of single feed or a sensor to keep the computational complexity as small as possible. The drifting phenomenon creates a virtual or a synthetic aperture array formed using a moving sensor. In this paper, we interchangeably use the terms single feed or single sensor.

Let the position vector of a single feed with respect to the earth center at time instant $t$ to be represented by,

$$
\mathbf{r}(t)=[x(t), \quad y(t), \quad z(t)]^{T},
$$

where, $\mathbf{r}(t) \in \mathbb{R}^{3 \times 1}$. The argument $t$ indicates the evolution of the array with time. Assume a narrow-band interferer present on the ground with two dimensional DOA $(\theta, \phi)$, and is constantly interfering the uplink transmission to the satellite, where $\theta$, and $\phi$ are the azimuth, and elevation angles, respectively. Satellite position measurements are calculated with respect to the earth center.

We assume the interfering signal $x(t) e^{j 2 \pi f t}$, centered at frequency $f$, transmitted at time instant $t$ from an interferer located in the far-field of the virtual array so that the plane wave assumption holds. Then, the interference signal sample collected at time instant $t_{i}$ is,

$$
y\left(t_{i}\right)=x\left(t_{i}-\tau_{i}\right) e^{j 2 \pi f\left(t_{i}-\tau_{i}\right)}+n\left(t_{i}\right), \forall i=1,2, \ldots, M,
$$

where $x(t)$ is some deterministic signal, $\tau_{i}$ is the time required by the signal to reach the sensor at $t_{i}$ time instant, $n\left(t_{i}\right) \in \mathbb{C}$ is the zero-mean complex Gaussian random process that is, $n\left(t_{i}\right) \sim \mathcal{C N}\left(0, \sigma^{2}\right) \forall i=1,2, \ldots, M$. The time of arrival, $\tau_{i}$ can be written as,

$$
\tau_{i}=\frac{\mathbf{r}\left(t_{i}\right)^{T} \mathbf{k}}{2 \pi f},
$$

where $\mathbf{r}\left(t_{i}\right)$ is the position vector of the sensor at $t_{i}$ time instant, and $\mathbf{k}$ denotes the wave number vector in the direction of the interfering signal,

$$
\mathbf{k}=\frac{2 \pi}{\lambda}\left[\begin{array}{ccc}
\cos \theta \cos \phi, \quad \sin \theta \cos \phi, \quad \sin \phi
\end{array}\right]^{T},
$$

where $\lambda$ is the wavelength corresponding to $f$. The downconverted signal at $t_{i}$ time instant can be written as,

$$
y\left(t_{i}\right)=x\left(t_{i}-\tau_{i}\right) e^{-j 2 \pi f \tau_{i}}+n\left(t_{i}\right) .
$$

After down-converting and collecting all the $M$ samples, we have the following signal model,

$$
\left[\begin{array}{c}
y\left(t_{1}\right) \\
y\left(t_{2}\right) \\
\vdots \\
y\left(t_{M}\right)
\end{array}\right] \approx x\left(t_{1}\right)\left[\begin{array}{c}
e^{-j \mathbf{r}\left(t_{1}\right)^{T} \mathbf{k}} \\
e^{-j \mathbf{r}\left(t_{2}\right)^{T} \mathbf{k}} \\
\vdots \\
e^{-j \mathbf{r}\left(t_{M}\right)^{T} \mathbf{k}}
\end{array}\right]+\left[\begin{array}{c}
n\left(t_{1}\right) \\
n\left(t_{2}\right) \\
\vdots \\
n\left(t_{M}\right)
\end{array}\right] .
$$

The typical carrier frequency range used in Ku-band is $9.75-$ $12.5 \mathrm{GHz}$ with bandwidth (BW) ranging in the interval $26-62$ $\mathrm{MHz}$. Because of the relatively small BW in comparison with the carrier frequency, the signal can be assumed to be narrowband. Therefore, without loss of generality we can assume the baseband signal $x(t)$ to be narrow-band and it follows from section 1.6 of [9], that $x\left(t_{i}-\tau_{i}\right) \approx x\left(t_{1}\right) \forall i=1,2, \ldots, M$. More compactly, we can write the above set of equations as,

$$
\mathbf{y}=x_{1} \mathbf{a}(\theta, \phi)+\mathbf{n},
$$

where $\mathbf{y} \in \mathbb{C}^{M \times 1}, x_{1}=x\left(t_{1}\right), \mathbf{a}(\theta, \phi) \in \mathbb{C}^{M \times 1}$ is known as the array response vector, and $\mathbf{n} \in \mathbb{C}^{M \times 1}$. We resort to this strong assumption to realize on-board localization. Alleviating this assumption will be a part of future work.

In practice, it may happen more than one interfering station can completely jam the uplink transmission, so the general system model considering $P$ narrow-band interfering signals each with frequency $f$ can be written as,

$$
\mathbf{y}=\mathbf{A}(\boldsymbol{\Theta}) \mathbf{x}+\mathbf{n},
$$

where $\mathbf{A}(\boldsymbol{\Theta}) \in \mathbb{C}^{M \times P}, \boldsymbol{\Theta}=\left[\theta_{1}, \phi_{1}, \theta_{2}, \phi_{2}, \ldots, \theta_{P}, \phi_{P}\right]^{T}$, $\mathbf{x} \in \mathbb{C}^{P \times 1}$, and again $\mathbf{n} \in \mathbb{C}^{M \times 1}$. Each entry of the vector $\mathbf{x}$ corresponds to the contribution from each interfering signal. Most of the subspace methods [10],[11],[12], operate on eigen 
structure of the covariance of the measurements which is given as,

$$
\mathbf{R}=E\left\{\mathbf{y} \mathbf{y}^{H}\right\}=\mathbf{A}(\boldsymbol{\Theta}) \mathbf{R}_{x x} \mathbf{A}(\boldsymbol{\Theta})^{H}+\sigma^{2} \mathbf{I},
$$

where $\mathbf{R} \in \mathbb{C}^{M \times M}$ is the covariance matrix of $\mathbf{y}, \mathbf{R}_{x x}$ is the covariance matrix of $\mathbf{x}$, and $\mathbf{I} \in \mathbb{R}^{M \times M}$ is an identity matrix. But in general the covariance matrix is estimated by the sample covariance matrix. Once, this sample covariance matrix is calculated, then high resolution subspace methods can be applied to estimate the DOAs.

\section{A. Partially known array manifold}

The aim is to estimate the unknown parameter vector $\Theta$ from the measurement vector $\mathbf{y}$. In a typical SATCOM architecture, satellite monitoring unit (SMU), tracks the position of the satellite at certain time instances, say intervals of $\Delta T$, which is of the order of few minutes. On the other hand, a very high sampling rate is envisaged on-board the satellite to digitize the incoming signal. Thus, equation (6) yields a high dimensional vector with sparsely known position vectors $\mathbf{r}\left(\mathbf{t}_{\mathbf{i}}\right)$ for the covariance estimation in equation (9). This leads to a scenario with partially known array manifold $\mathbf{A}(\boldsymbol{\Theta})$ in equation (9). However, most of the DOA estimation techniques like multiple signal classification (MUSIC), rely on searching over the whole field of view (FOV), thus necessitating the information about the entire array manifold. Additionally, the scenario provides a single snapshot leading to estimation inaccuracies of $\mathbf{R}$. In the next section, we provide a methodology and a potential solution to these problems.

\section{ON-BOARD INTERFERENCE LOCALIZATION}

In the preceding section, we stated the objective of estimating the DOAs associated with each interfering signal on-board the satellite. Standard subspace methods for DOA estimation relies on multiple snapshots to construct the sample covariance matrix, but we are limited by only one available snapshot. Also, for many measurements, the position vector of the sensor position is not known to the SMU. That is if we consider the following matrix $\mathbf{R}_{\mathrm{pos}} \in \mathbb{R}^{3 \times M}$ consisting of sensor positions at all time instants,

$$
\mathbf{R}_{\mathrm{pos}}=\left[\mathbf{r}\left(t_{1}\right), \mathbf{r}\left(t_{2}\right), \ldots, \mathbf{r}\left(t_{M}\right)\right] .
$$

Many position vectors in the above matrix are not known to the GW or SMU. In other words, only a subset $M_{1} \subset M$ is known to the SMU, which makes this problem more difficult. But satellite continues to receive signals. Therefore, on-board measurements are available, but their corresponding position vectors are not known. The next problem is that the highresolution subspace methods cannot be directly employed because of the availability of single snapshot. In the following subsections, we answer the aforementioned questions and provide potential heuristic solutions to these problems. Investigation of more advanced solutions is a subject of future works.

\section{A. Predicting missing sensor positions}

It is known that after certain time intervals SMU keeps track of the satellite position, along with the information about its instantaneous velocity. So, we propose to use appropriate 3D curve interpolation techniques with appropriate resolution, which can predict the trajectory of the satellite based on the position vectors available to the SMU. Thus, what we essentially have on-board is a very high dimensional measurement vector y. Hence, one of the challenges associated, that is unknown satellite positions with the signal model can be alleviated.

\section{B. Mapping single snapshot to multiple snapshots}

The next step is to estimate the DOAs. As already stated we want to use standard high-resolution subspace methods to achieve the desired localization accuracy. But most of these techniques rely on the efficient estimation of the eigenstructure of the covariance matrix of the measurements. A typical approximation to the covariance matrix is to use the sample covariance matrix, which again requires a large number of multiple measurements. Therefore, we propose to create multiple snapshots from a single snapshot. Because of high rate sampling, the measurements sampled near a small neighbourhood of a position can be approximated to be received from the position itself. In other words, we can treat measurements corresponding to a small vicinity of the known sensor positions as if they belong to them.

Therefore, we define a mapping $g: \mathbf{y} \rightarrow \mathbf{Y}$, where $\mathbf{y} \in \mathbb{C}^{M \times 1}$, and $\mathbf{Y} \in M^{\prime} \times N^{\prime}$. This mapping selects a subset of the measurement vector $\mathbf{y}$ to calculate the sample covariance matrix. For example, suppose we have $M=10^{6}$ and we divide this vector into 10 segments. That is we choose $M^{\prime}=11$ virtual sensor positions and each segment will have $10^{5}$ measurements. Now, the next step is to find an appropriate number of snapshots, $N^{\prime}$, required to estimate the covariance matrix such that DOAs can be estimated with the desired accuracy. This transformation essentially builds up the rank of the covariance matrix which helps in identifying more than one source using single snapshot. This idea is similar to spatial smoothing using single snapshot [13], but in spatial smoothing, we generally focus on overlapped sub-arrays to build the rank of the covariance. But the essence of this transformation is to create multiple snapshots to be able to localize more than one source.

\section{Estimating DOAs}

In order to get the multiple snapshots from a single snapshot, we need to look at the trade-off between the $M^{\prime}$, and $N^{\prime}$. Because increasing $M^{\prime}$ would increase the effective aperture, so that more closely spaced sources can be localized, and increasing $N^{\prime}$, the number of snapshots, provides a better approximation to the sample covariance matrix. But increasing $N^{\prime}$ beyond certain threshold also losses the structure similar to ULA around each sensor position known to the SMU, thus estimation accuracy may degrade. To get some reasonable value for these two parameters, in the next section we provide 
estimation error analysis, based on that we select these parameters. Once we have the multiple snapshots, we can calculate the sample covariance matrix as,

$$
\mathbf{R}=\frac{1}{N^{\prime}} \sum_{k=1}^{N^{\prime}} \mathbf{y}_{k} \mathbf{y}_{k}^{H},
$$

where $\mathbf{y}_{k}$ is the $k$-th column of the measurement matrix $\mathbf{Y}$. After calculating the sample covariance, we employ multiple signal classification (MUSIC) algorithm [14], [15] to estimate the DOAs. MUSIC works on the eigendecomposition of the covariance matrix, thereby identifying the noise subspace corresponding to smaller eigenvalues, and then testing the orthogonality of the array steering vectors onto the noise subspace. Because of the orthogonality between the signal subspace spanned by the columns of the matrix $\mathbf{A}(\boldsymbol{\Theta})$ and the noise subspace spanned by the columns of matrix $\mathbf{G}$, we have the following 2-D search to estimate the DOAs,

$$
\begin{aligned}
\{\widehat{\theta}, \widehat{\phi}\}=\arg \min _{\{\theta, \phi\}} \mathbf{a}(\theta, \phi)^{H} \mathbf{G G}^{H} \mathbf{a}(\theta, \phi) & \\
\text { subject to } \quad & \theta \in \boldsymbol{\theta}^{\prime}=\left\{\theta_{1}^{\prime}, \theta_{2}^{\prime}, \ldots, \theta_{K 1}^{\prime}\right\}, \\
\phi \in \phi^{\prime} & =\left\{\phi_{1}^{\prime}, \phi_{2}^{\prime}, \ldots, \phi_{K 2}^{\prime}\right\},
\end{aligned}
$$

where $\boldsymbol{\theta}^{\prime}, \boldsymbol{\phi}^{\prime}$ are the fine grids for azimuth, elevation angles respectively. The grid resolution depends on the accuracy desired for the DOA estimation. The above optimization is the 2-D extension of the MUSIC algorithm [14], [15] and can be carried out on-ground due to the $O\left(n^{2}\right)$ complexity involved in 2-D search.

\section{Simulation Results}

In this section, we provide numerical simulation results to get an approximate value of $M^{\prime}$, and $N^{\prime}$. Based on realistic parameter settings we consider a moving feed scenario observing two closely spaced narrow-band interfering signals at center frequency $f=2 \mathrm{GHz}$, constantly interfering the uplink transmission with DOAs (azimuth,elevation): $\left(60^{\circ}, 62^{\circ}\right)$, and $\left(60.5^{\circ}, 62^{\circ}\right)$. Because this application demands low interference to noise ratio (INR), we present the simulation results at an INR of $0 \mathrm{~dB}$. Each entry of the vector $\mathbf{x}$ is modeled using baseband modulation with binary phase shift keying (BPSK) signaling. Suppose that we know the satellite position at $M_{1}$ instants, then in order to predict the trajectory, we use spline-based curve interpolation to get the missing position vectors. The data corresponding to the coarse position vectors of the satellite was based on data provided by the operator SES. We have used five position vectors from the data provided. Interpolation is done with a dense sampling grid of 5 Million points. Then, we generate the measurement vector y using the signal model of equation (8). Having generated the snapshot, the next step is to create multiple snapshots using this very high dimensional vector. This vector is then divided into $M^{\prime}=200$ segments, and then taking $N^{\prime}=25000$ measurements in each segment, and thus creating a multiple snapshot matrix $\mathbf{Y} \in \mathbb{C}^{M^{\prime} \times N^{\prime}}$. Now, the sample covariance is

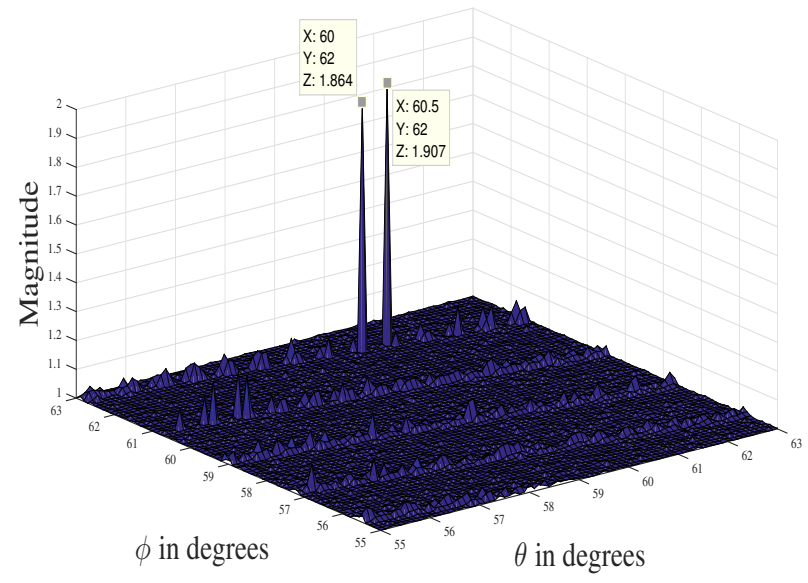

Fig. 1. Estimated DOAs using the MUSIC algorithm, distinctly resolving the two interference signals.

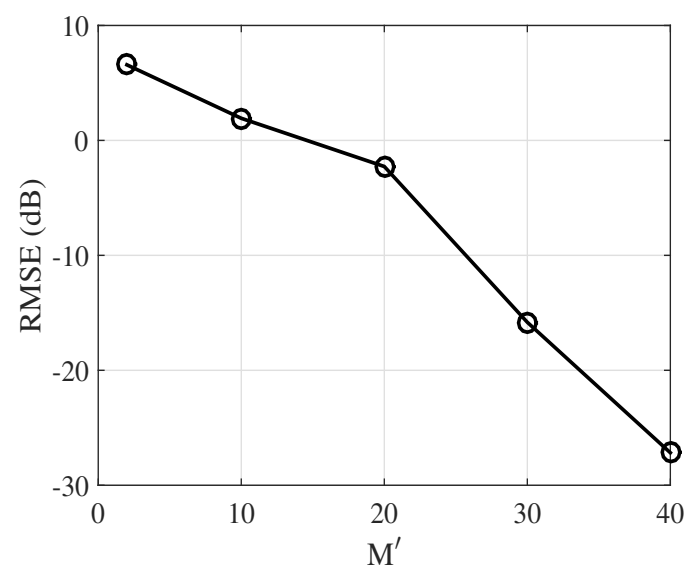

Fig. 2. Estimation error (in dB) perofrmance of the MUSIC algorithm with number of sensor positions with a constant budget of $M^{\prime} N^{\prime}=5000$.

calculated to employ MUSIC algorithm. Figure 1 shows the MUSIC spectrum indicating the detection of correct DOAs.

Now, we look at the complexity-performance trade-off. We define a product $M^{\prime} N^{\prime}=C$, where $C$ is some constant and it defines a fixed budget on the number of measurements. Here, we have used $C=5000$. Generally, used metric to study the estimation error performance of an algorithm is the root mean square error (RMSE). The numerical simulation result for the estimation error variation with $M^{\prime}$ in the parameter vector $\Theta$, averaged over 5000 Monte-Carlo runs is shown in figure 2. It is clearly shown in figure 2 that the estimation error decreases as the number of sensors increases. For $M^{\prime}=40$ there is a substantial difference in the estimation error compared with $M^{\prime}=30$. But for $M^{\prime}=40$ computational complexity in finding the DOAs is large compared to $M^{\prime}=30$. Thus, we can choose the number of sensor positions based on the desired estimation accuracy and the performance-complexity trade-off. 


\section{CONCLUSION}

In this paper we provide a methodology to localize closely spaced narrow-band interferers using a single moving feed with single snapshot on-board the satellite. Proposed method has been verified from numerical simulations. To find a good choice of $M^{\prime}$, and $N^{\prime}$, we provided numerical simulation results for minimizing the estimation error in LS sense averaged over several Monte-Carlo runs. The choice of $M^{\prime}$, and $N^{\prime}$ is based on the performance-complexity trade-off. This choice can be used for resolving the two closely spaced interfering sources accurately. This scheme can be possibly implemented into two parts, namely on-board: construction of covariance matrix, and on-ground: final interference localization based on the covariance matrix constructed on-board the satellite using subspace high resolution techniques like MUSIC, subspace fitting etc. In this work we have assumed the $0.5^{\circ}$ resolution between two interference signals, algorithms providing $0.1^{\circ}$ resolution is a part of future work.

\section{ACKNOWLEDGEMENT}

This work is supported by the National Research Fund (FNR), Luxembourg under the AFR-PPP grant for Ph.D. project (Reference: 11607283) on "Power Efficient Sparse Signal Processing for Digital Wideband On-Board Techniques" (SPASAT), and the CORE-PPP project on "On-Board Processing Techniques for High Throughput Satellites" (PROSAT).

\section{REFERENCES}

[1] "Inclined orbit operation of geostationary satellites." [Online]. Available: http://www.satsig.net/satellite/inclined-orbit-operation.htm

[2] "Siemens. one-satellite geolocation system - beyond the limits of traditional satellite interference localization." [Online]. Available: https://atos.net/en/convergence-creators/portfolio/siecams/ siecams-ils-one?utm_source=www.convergence-creators.siemens.com/ siecams-ils-one1.html\&utm_medium $=301$

[3] "Tackling vsat interference." [Online]. Available: https://artes.esa.int/ news/tackling-vsat-interference

[4] D. Ho, J. Chu, and M. Downey, "Determining a geolocation solution of an emitter on earth using satellite signals," Feb. 23 2010, uS Patent 7,667,640. [Online]. Available: http://www.google.com.na/ patents/US7667640

[5] A. Kalantari, S. Maleki, S. Chatzinotas, and B. Ottersten, "Frequency of arrival-based interference localization using a single satellite," in 2016 8th Advanced Satellite Multimedia Systems Conference and the 14th Signal Processing for Space Communications Workshop (ASMS/SPSC), Sept 2016, pp. 1-6.

[6] D. Ho, J. Chu, and M. Downey, "Determining transmit location of an emitter using a single geostationary satellite," Jun. 11 2013, uS Patent 8,462,044. [Online]. Available: https://www.google.com/patents/ US8462044

[7] S. Borissov, Y. Wu, and D. Mortari, "Eastwest GEO Satellite Station-Keeping with Degraded Thruster Response," Aerospace, vol. 2, no. 4, pp. 581-601, 2015. [Online]. Available: http: //www.mdpi.com/2226-4310/2/4/581

[8] E. Soop, Handbook of Geostationary Orbits, ser. Space Technology Library. Netherlands: Springer, 1994, vol. 3.

[9] A. Manikas, Differential Geometry in Array Processing. Imperial College Press, 2004.

[10] A. L. Swindlehurst and T. Kailath, "A performance analysis of subspacebased methods in the presence of model errors. I. The MUSIC algorithm," IEEE Transactions on Signal Processing, vol. 40, no. 7, pp. 1758-1774, Jul 1992.

[11] M. Viberg, B. Ottersten, and T. Kailath, "Detection and estimation in sensor arrays using weighted subspace fitting," IEEE Transactions on Signal Processing, vol. 39, no. 11, pp. 2436-2449, Nov 1991.
[12] B. Ottersten, M. Viberg, and T. Kailath, "Analysis of subspace fitting and ML techniques for parameter estimation from sensor array data," IEEE Transactions on Signal Processing, vol. 40, no. 3, pp. 590-600, Mar 1992.

[13] A. Thakre, M. Haardt, and K. Giridhar, "Single snapshot spatial smoothing with improved effective array aperture," IEEE Signal Processing Letters, vol. 16, no. 6, pp. 505-508, June 2009.

[14] R. Schmidt, "Multiple emitter location and signal parameter estimation," IEEE Transactions on Antennas and Propagation, vol. 34, no. 3, pp. 276-280, Mar 1986.

[15] P. Stoica and A. Nehorai, "MUSIC, Maximum Likelihood, and CramerRao bound," IEEE Transactions on Acoustics, Speech, and Signal Processing, vol. 37, no. 5, pp. 720-741, May 1989. 